\title{
On dominance and minuscule Weyl group elements
}

\author{
Qëndrim R. Gashi · Travis Schedler
}

Received: 24 September 2009 / Accepted: 7 July 2010 / Published online: 10 August 2010

(C) Springer Science+Business Media, LLC 2010

\begin{abstract}
Fix a Dynkin graph and let $\lambda$ be a coweight. When does there exist an element $w$ of the corresponding Weyl group such that $w$ is $\lambda$-minuscule and $w(\lambda)$ is dominant? We answer this question for general Coxeter groups. We express and prove these results using a variant of Mozes' game of numbers.
\end{abstract}

Keywords Dominant weights · Minuscule Weyl group elements · Numbers game with a cutoff

\section{Introduction}

Mazur's Inequality $[17,18]$ is an important $p$-adic estimate of the number of rational points of certain varieties over finite fields. It can be formulated in purely group-theoretic terms, and the classical version can be viewed as a statement for the group $G L_{n}$ (see [15]). Kottwitz and Rapoport formulated a converse to this inequality [16], which is also related to the non-emptiness of certain affine Deligne-Lusztig varieties, and they reduced the proof to a purely root-theoretic problem, which is solved in [12]. A crucial step in [12] involves the use of Theorem 1.1 below, which we state after recalling some standard notation and terminology.

Q.R. Gashi (凶)

IHES, Bures-sur-Yvette, France

e-mail: qendrim@math.uchicago.edu

T. Schedler

MIT, Cambridge, USA

e-mail: trasched@math.mit.edu 
Let $\Gamma$ be a simply-laced Dynkin graph, ${ }^{1}$ with corresponding simple roots $\alpha_{1}, \ldots, \alpha_{n}$, positive roots $\Delta_{+}$, Weyl group $W$, and simple reflections $s_{1}, \ldots, s_{n} \in W$. Let $P_{\Gamma}$ be the lattice of coweights corresponding to $\Gamma$. Following Peterson, for $\lambda \in P_{\Gamma}$ and $w \in W$, we say that $w$ is $\lambda$-minuscule if there exists a reduced expression $w=s_{i_{1}} s_{i_{2}} \cdots s_{i_{t}}$ such that

$$
s_{i_{r}} s_{i_{r+1}} \cdots s_{i_{t}} \lambda=\lambda+\alpha_{i_{r}}^{\vee}+\alpha_{i_{r+1}}^{\vee}+\cdots+\alpha_{i_{t}}^{\vee}, \quad \forall r \in\{1,2, \ldots, t\},
$$

where $\alpha_{i}^{\vee} \in P_{\Gamma}$ is the simple coroot corresponding to $\alpha_{i}$. Equivalently (cf. [22]), a reduced product $w=s_{1} s_{2} \cdots s_{i_{t}}$ is $\lambda$-minuscule if and only if $\left\langle\lambda, \alpha_{i_{t}}^{\vee}\right\rangle=-1$ as well as $\left\langle s_{i_{r+1}} \cdots s_{i_{t}} \lambda, \alpha_{i_{r}}^{\vee}\right\rangle=-1$, for all $r \in\{1, \ldots, t-1\}$, where $\langle$,$\rangle is the Cartan pairing.$

Recall that an element $\mu \in P_{\Gamma}$ is called dominant if $\left\langle\mu, \alpha_{i}^{\vee}\right\rangle \geq 0, \forall i=1, \ldots, n$.

Theorem 1.1 For $\lambda \in P_{\Gamma}$, there exists a $\lambda$-minuscule element $w \in W$ such that $w(\lambda)$ is dominant if and only if

$$
\left\langle\lambda, \alpha^{\vee}\right\rangle \geq-1, \quad \forall \alpha \in \Delta_{+}
$$

The proof of this theorem is straightforward, and is given in Sect. 3. We also generalize the result to the case of extended Dynkin graphs, in the following manner. Let $\widetilde{\Gamma}$ be a simply-laced extended Dynkin graph, $\widetilde{W}$ be its Weyl group, and $R_{\widetilde{\Gamma}}$ be the root lattice, i.e., the span of the simple roots $\alpha_{i}$. Let $\widetilde{\Delta}_{+} \subset R_{\widetilde{\Gamma}}$ be the set of positive real roots (i.e., positive-integral combinations $\alpha$ of simple roots such that $\langle\alpha, \alpha\rangle=$ 2). Define $P_{\widetilde{\Gamma}}$ in this case to be the dual to the root lattice $R_{\widetilde{\Gamma}}$. Given $\alpha \in R_{\widetilde{\Gamma}}$ and $\lambda \in P_{\widetilde{\Gamma}}$, denote their pairing by $\alpha \cdot \lambda$. Let $\delta \in R_{\widetilde{\Gamma}}$ be the positive-integral combination of simple roots which generates the kernel of the Cartan form on $R_{\widetilde{\Gamma}}$. Finally, for $\alpha \in \widetilde{\Delta}_{+}$, let $\alpha^{\vee} \in P_{\widetilde{\Gamma}}$ be the element such that $\beta \cdot \alpha^{\vee}=\langle\beta, \alpha\rangle$ for all $\beta \in \widetilde{\Delta}_{+}$. Then, the notion of $\lambda$-minusculity carries over to this setting.

Theorem 1.2 For nonzero $\lambda \in P_{\widetilde{\Gamma}}$, there exists a $\lambda$-minuscule element $w \in \widetilde{W}$ such that $w(\lambda)$ is dominant if and only if

(i) $\alpha \cdot \lambda \geq-1, \quad \forall \alpha \in \widetilde{\Delta}_{+}, \quad$ and

(ii) $\delta \cdot \lambda \neq 0$.

We generalize the theorems above in two directions. First, we allow $\lambda$ to be nonintegral, i.e., to lie in $P_{\Gamma} \otimes_{\mathbb{Z}} \mathbb{R}$ (respectively $P_{\widetilde{\Gamma}} \otimes_{\mathbb{Z}} \mathbb{R}$ ) and not just in $P_{\Gamma}$ (respectively $P_{\widetilde{\Gamma}}$ ). Second, we consider all Coxeter groups, not just finite and affine ones. For example, in the first direction, if $\lambda \in P_{\Gamma} \otimes_{\mathbb{Z}} \mathbb{R}$, the notion of $\lambda$-minuscule Weyl group element should be generalized accordingly: $w \in W$ is $\lambda$-minuscule if there exists a reduced expression $w=s_{i_{1}} \cdots s_{i_{t}}$ such that $s_{i_{r}} \cdots s_{i_{t}} \lambda=\lambda+\xi_{r} \alpha_{i_{r}}^{\vee}+\cdots+\xi_{t} \alpha_{i_{t}}^{\vee}$ for all $r \in\{1, \ldots, t\}$, for some positive real numbers $\xi_{1}, \ldots, \xi_{t} \leq 1$.

In the original situation (for $\lambda \in P_{\Gamma}$ "integral" and $\Gamma$ Dynkin), we prove a stronger result:

\footnotetext{
${ }^{1}$ No information is lost in thinking of a simply-laced Dynkin diagram as an undirected graph, and so we do so throughout. In Sect. 5, we consider non-simply-laced diagrams, which we will consider as undirected graphs together with additional data.
} 
Theorem 1.3 Under the assumptions of Theorem 1.1, there exists a $\lambda$-minuscule element $w \in W$ such that $w(\lambda)$ is dominant if and only if

(i) $\left\langle\lambda, \alpha_{i}^{\vee}\right\rangle \geq-1$ for every simple root $\alpha_{i}$, and

(ii) For every connected subgraph $\Gamma^{\prime} \subseteq \Gamma$, the restriction $\left.\lambda\right|_{\Gamma^{\prime}}$ is not a negative coroot.

In the theorem, the restriction $\left.\lambda\right|_{\Gamma^{\prime}} \in P_{\Gamma^{\prime}}$ is the unique element such that $\left\langle\left.\lambda\right|_{\Gamma^{\prime}}, \alpha_{i}^{\vee}\right\rangle=\left\langle\lambda, \alpha_{i}^{\vee}\right\rangle$ for all simple roots $\alpha_{i}$ associated to the vertices of $\Gamma^{\prime}$.

We also prove a similar result for extended Dynkin graphs (see Theorem 4.1), and generalize it so as to include the case where $\lambda$ lies in a finite Weyl orbit.

Remark 1.4 Condition (1.1) is equivalent to the non-negativity of the coefficients of Lusztig's $q$-analogues of weight multiplicity polynomials (see [3, Theorem 2.4]). It is also equivalent to the vanishing of the higher cohomology groups of the line bundle that corresponds to $\lambda$ on the cotangent bundle of the flag variety (op. cit.). We hope to address and apply this in future work.

The paper is organized as follows. The second section introduces the terminology of Mozes' game of numbers [19] and its variant with a cutoff [12], which provides a useful language to state and prove our results. We also recall some preliminaries on Dynkin and extended Dynkin graphs. In the third section we solve the numbers game with a cutoff for Dynkin and extended Dynkin graphs (Theorem 3.1), in particular proving Theorems 1.1 and 1.2 and the non-integral versions thereof. Next, in Sect. 4, we give a more explicit solution in the integral case, which proves Theorem 1.3 and the corresponding result for extended Dynkin graphs. In the last section, we generalize Theorem 1.1 to the case of arbitrary Coxeter groups.

Convention In Sects. 3, 4, and 5, all the graphs we consider will be simply-laced Dynkin and extended Dynkin graphs (i.e., of types ADE and $\widetilde{A} \widetilde{D} \widetilde{E}$ ). We generalize our results to non-simply-laced diagrams in Sect. 5.

\section{The numbers game with and without a cutoff}

In this section we introduce the numbers game with a cutoff, which provides a useful language to state our results. We begin with some preliminaries on Dynkin and extended Dynkin graphs.

\subsection{Preliminaries on Dynkin and extended Dynkin graphs}

As was mentioned in the introduction, in this section as well as in the next two, we will largely restrict our attention to simply-laced Dynkin and extended Dynkin graphs. By this, we mean graphs of type $A_{n}, D_{n}$, or $E_{n}$, or $\tilde{A}_{n}, \tilde{D}_{n}$, or $\tilde{E}_{n}$. 
For such a graph $\Gamma$, let $\Delta$ be the set of (real) ${ }^{2}$ roots of the associated root system, and $\Delta_{+}$the set of positive roots. Let $I$ denote its set of vertices, so that $\alpha_{i}$ are the simple roots for $i \in I$, and let $\omega_{i}$ be the corresponding fundamental coweights. Identify $\mathbb{Z}^{I}$ with the root lattice (i.e., the integral span of the $\alpha_{i}$ ), so that $\Delta \subseteq \mathbb{Z}^{I}$, and $\alpha_{i} \in \mathbb{Z}^{I}$ are the elementary vectors. Although we will use subscripts (e.g., $\beta_{i}$ of $\beta \in \mathbb{Z}^{I}$ ) to denote coordinates, we will never use them for a vector denoted by $\alpha$ or $\omega$, to avoid confusion with the simple roots $\alpha_{i}$ and the fundamental coweights $\omega_{i}$.

We briefly recall the essential facts about $\Delta_{+}$and $\Delta$. We have $\Delta=\Delta_{+} \sqcup\left(-\Delta_{+}\right)$, and $\Delta_{+}=\left\{\alpha \in \mathbb{Z}_{\geq 0}^{I}:\langle\alpha, \alpha\rangle=2\right\}$, where $\langle$,$\rangle is the Cartan form$

$$
\left\langle\alpha_{i}, \alpha_{j}\right\rangle= \begin{cases}2, & \text { if } i=j, \\ -1, & \text { if } i \text { is adjacent to } j, \\ 0, & \text { otherwise, }\end{cases}
$$

which is positive-definite in the Dynkin case and positive-semidefinite in the extended Dynkin case. It is well known that $\Delta_{+}$is finite in the Dynkin case. Consider the extended Dynkin case, and let us switch notation to $\widetilde{\Gamma}, \widetilde{\Delta}, \widetilde{\Delta}_{+}$, and $\widetilde{I}$. We may write $\widetilde{\Gamma} \supsetneq \Gamma$ where $\Gamma$ is the Dynkin graph of corresponding type. The vertex $i_{0}=\widetilde{I} \backslash I$ is called an extending vertex (the other extending vertices being obtained as the complements of different choices of $\Gamma$ ). Let $\Delta_{+}$the set of positive roots for $\Gamma$. There is an inclusion $\Delta_{+} \subset \widetilde{\Delta}_{+}$obtained by setting the coefficient at $i_{0}$ to zero, and $\widetilde{\Delta}_{+}=\left(\Delta_{+}+\mathbb{Z}_{\geq 0} \delta\right) \sqcup\left(-\Delta_{+}+\mathbb{Z}_{>0} \delta\right)$, for the unique vector $\delta \in \mathbb{Z}_{>0}^{\widetilde{I}}$ characterized by $\langle\delta, u\rangle=0$ for all $u \in \mathbb{R}^{\tilde{I}}$ and $\delta_{i_{0}}=1$.

Switching back to $\Gamma, \Delta_{+}$, and $I$, for either the Dynkin or extended Dynkin case, we recall the simple reflections. For any vertex $i \in I$, let $s_{i}: \mathbb{R}^{I} \rightarrow \mathbb{R}^{I}$ be defined by $s_{i}(\beta)=\beta-\left\langle\beta, \alpha_{i}\right\rangle \alpha_{i}$. It is well known that $\beta \in \Delta_{+}$implies $s_{i}(\beta) \in \Delta_{+}$unless $\beta=\alpha_{i}$, in which case $s_{i}\left(\alpha_{i}\right)=-\alpha_{i}$. Also, $s_{i}(\delta)=\delta$ for all $i$.

For any $\beta \in \Delta_{+}$, its height, $h(\beta)$, is defined as $h(\beta)=\sum_{i \in I} \beta_{i}$, where $\beta=\left(\beta_{i}\right)=$ $\sum_{i} \beta_{i} \alpha_{i}$. Note that $\beta$ may be obtained from some simple root $\alpha_{i}$ by applying $h(\beta)-1$ simple reflections, and is not obtainable from any simple root by applying fewer simple reflections.

\subsection{The numbers game with and without a cutoff}

We first recall Mozes' numbers game [19]. Fix an unoriented, finite graph with no loops and no multiple edges. (For the generalized version of this game, with multiplicities, see Sect. 5.) Let $I$ be the set of vertices. The configurations of the game consist of vectors $\mathbb{R}^{I}$. The moves of the game are as follows: For any vector $v \in \mathbb{R}^{I}$ and any vertex $i \in I$ such that $v_{i}<0$, one may perform the following move, called firing the vertex $i: v$ is replaced by the new configuration $f_{i}(v)$, defined by

$$
f_{i}(v)_{j}= \begin{cases}-v_{i}, & \text { if } j=i, \\ v_{j}+v_{i}, & \text { if } j \text { is adjacent to } i, \\ v_{j}, & \text { otherwise. }\end{cases}
$$

\footnotetext{
${ }^{2}$ These are sometimes called "real roots" in the literature to exclude multiples of the so-called imaginary root $\delta$ below, which are also roots of the associated Kac-Moody algebra. We will omit the adjective "real."
} 


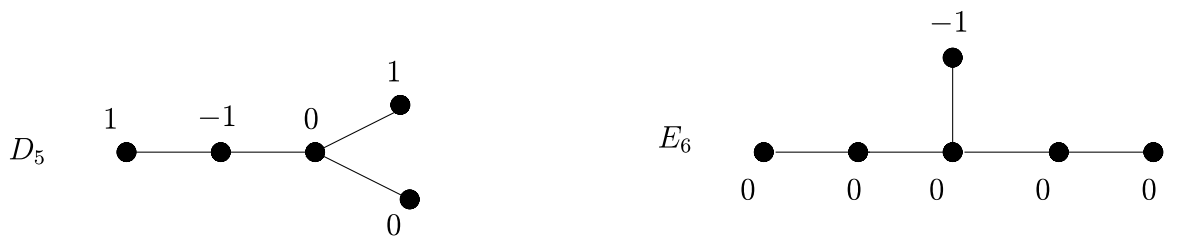

Fig. 1 Examples of winning $\left(D_{5}\right)$ and losing $\left(E_{6}\right)$ configurations

The entries $v_{i}$ of the vector $v$ are called amplitudes. The game terminates if all the amplitudes are nonnegative. Let us emphasize that only negative-amplitude vertices may be fired. ${ }^{3}$

In [11], the numbers game with a cutoff was defined: The moves are the same as in the ordinary numbers game, but the game continues (and in fact starts) only as long as all amplitudes remain greater than or equal to -1 . Such configurations are called allowed. Every configuration which does not have this property is called forbidden, and upon reaching such a configuration the game terminates (we lose). We call a configuration winning if it is possible, by playing the numbers game with a cutoff, to reach a configuration with all nonnegative amplitudes.

Call a configuration losing if, no matter how the game is played, one reaches a forbidden configuration. By definition, any losing configuration remains so by playing the numbers game. We will see that the same is true for winning configurations (Corollary 5.2).

We now explain how to interpret the results from the introduction in terms of this language. Let $\Gamma$ be a Dynkin graph, with set of vertices $I$. To every element $\lambda \in P_{\Gamma}$ one can associate naturally an integral configuration of $\Gamma$, still denoted by $\lambda$, where the amplitude corresponding to the vertex $\alpha_{i}$ is given by $\left\langle\lambda, \alpha_{i}^{\vee}\right\rangle$. Firing the vertex $\alpha_{j}$ changes these amplitudes to $\left\langle s_{j}(\lambda), \alpha_{i}^{\vee}\right\rangle$, i.e., gives the natural configuration (on the vertices of $\Gamma$ ) associated to the simple reflection $s_{j}(\lambda)$ of $\lambda$. In other words, using the identifications made in the previous subsection between the coroot space and $\mathbb{Z}^{I}$, and letting $\cdot$ denote the standard dot product on $\mathbb{R}^{I}$, we have

$$
s_{i}(\alpha) \cdot v=\alpha \cdot f_{i}(v), \quad s_{i}(\alpha) \cdot f_{i}(v)=\alpha \cdot v,
$$

for any configuration $v$. In terms of Lie theory, we may think of the $s_{i}$ as acting on $\mathbb{R}^{I}$ with basis given by the simple roots, and the $f_{i}$ as acting on the dual $\mathbb{R}^{I}$, with basis given by the fundamental coweights. (Formula (2.2) remains true in the case of extended Dynkin graphs.)

The existence of an element $w \in W$ such that $w(\lambda)$ is dominant is then equivalent to the winnability of the usual numbers game with initial configuration $\lambda$ (and hence, one always wins). Of course, we want to impose the extra condition that $w$ be $\lambda$-minuscule, which is equivalent to imposing the -1 cutoff to the numbers game. Thus, Theorem 1.1 gives a characterization of the winning configurations $v \in \mathbb{Z}^{I}$ for the numbers game with a cutoff, where $v_{i}=\left\langle\lambda, \alpha_{i}^{\vee}\right\rangle, \lambda \in P_{\Gamma}$, and the graph $\Gamma$ is a

\footnotetext{
${ }^{3}$ In some of the literature, the opposite convention is used, i.e., only positive-amplitude vertices may be fired.
} 
Dynkin one. Later on, we will give similar descriptions in terms of the numbers game with a cutoff for the other results stated in the introduction.

Note that in the paragraph above we only considered the case of integral $\lambda$, but the analogy holds in the non-integral case as well, and now we study the winnability of the numbers game with a cutoff with real amplitudes, where we may fire any vertex with amplitudes from $[-1,0)$ and not just those with amplitude -1 as in the integral case.

Example 2.1 In Fig. 1, in the case of $\Gamma=D_{5}$, we have $\left\langle\lambda, \alpha_{i}^{\vee}\right\rangle=v_{i}$, with $v_{1}=1$, $v_{2}=-1, v_{3}=0, v_{4}=1$, and $v_{5}=0$, where we have labeled the vertices of the graph as in [2], Plate IV, p. 271. Therefore, if we write $\lambda$ in the basis of the fundamental coweights $\left(\omega_{i}\right)_{i=1, \ldots, 5}$, we find that $\lambda=\omega_{1}-\omega_{2}+\omega_{4}$. It can easily be seen that $w=$ $s_{5} s_{3} s_{2}$ is $\lambda$-minuscule and $w(\lambda)=\omega_{5}$, so $w(\lambda)$ is dominant. The simple reflections $s_{5}, s_{3}$, and $s_{2}$ correspond to the firing (in the reverse order) of the corresponding vertices of the Dynkin graph.

Continuing with Fig. 1 , for $\Gamma=E_{6}$, we see that in this case $\lambda=-\omega_{2}$, but there exists no $w \in W$ such that both $w$ is $\lambda$-minuscule and $w(\lambda)$ is dominant, since the numbers game with a cutoff is losing for the configuration $\left(\left\langle\lambda, \alpha_{i}^{\vee}\right\rangle\right)_{i \in I}$. Here we have used the same labeling of vertices of $\Gamma$ as in [2], Plate V, p. 276.

The language of the numbers game with a cutoff is useful because it makes apparent certain phenomena that already occur without the bound of -1 or indeed with a different bound. It also allows one to use results from the usual Mozes' numbers game, which has been widely studied (cf. [4-10, 20, 21, 23, 24]), ${ }^{4}$ and yields useful algorithms for computing with the root systems and reflection representations of Coxeter groups (see [1, Sect. 4.3] for a brief summary).

Finally, we recall some basic results about the usual numbers game, and why it exhibits special behavior in the Dynkin and extended Dynkin cases:

\section{Proposition 2.2}

(i) [19] If the usual numbers game terminates, then it must terminate in the same number of moves and at the same configuration regardless of how it is played.

(ii) In the Dynkin case, the usual numbers game must terminate.

(iii) [7] In the extended Dynkin case, for $v \neq 0$, the usual numbers game terminates if and only if $\delta \cdot v>0$.

(iv) [7] Whenever the usual numbers game does not terminate, it reaches infinitely many distinct configurations, except for the case of an extended Dynkin graph where $\delta \cdot v=0$, in which case only finitely many configurations are reached (i.e., the game "loops"). 5

Thus, provided we can determine which configurations are winning (for the numbers game with a cutoff) in the Dynkin case and the extended Dynkin case, then with

\footnotetext{
${ }^{4}$ Mozes' numbers game originated from (and generalizes) a 1986 IMO problem.

${ }^{5}$ Stronger results were stated in [7], and a detailed study appears in [13].
} 
the additional condition $\delta \cdot v>0$, these and $v=0$ are also the ones that terminate in a nonnegative configuration, and this configuration (and the number of moves required to get there) is unique.

\section{The (extended) Dynkin case}

Theorem 3.1 In the Dynkin case, a configuration $v \in \mathbb{R}^{I}$ is winning if and only if

$$
\alpha \cdot v \geq-1, \quad \forall \alpha \in \Delta_{+} .
$$

Otherwise, $v$ is losing.

In the extended Dynkin case, $v \neq 0$ is winning if and only if both

$$
\alpha \cdot v \geq-1, \quad \forall \alpha \in \widetilde{\Delta}_{+}
$$

and $\delta \cdot v \neq 0$. If (3.2) is satisfied but $\delta \cdot v=0$ (and $v \neq 0$ ), then $v$ is looping and the game cannot terminate. Finally, if (3.2) is not satisfied (e.g., if $\delta \cdot v<0)$, then $v$ is losing.

Remark 3.2 Theorem 3.1 implies Theorems 1.1 and 1.2, as well as their "nonintegral" versions.

The above theorem shows, in particular, that exactly one of the following is true: $v$ is winning, looping, or losing. If we return to Example 2.1 (see also Fig. 1), we see that in the case of the graph of type $E_{6}$ the coweight $\lambda=-\omega_{2}$ is such that when paired with the coroot $\alpha_{1}^{\vee}+2 \alpha_{2}^{\vee}+2 \alpha_{3}^{\vee}+3 \alpha_{4}^{\vee}+2 \alpha_{5}^{\vee}+\alpha_{6}^{\vee}$ we get -2 , so the corresponding configuration is losing, as we have noted already.

To prove the theorem, it is helpful to introduce the set

$$
X_{v}:=\left\{(\alpha, \alpha \cdot v) \mid \alpha \in \Delta_{+}, \alpha \cdot v<0\right\} .
$$

Consider the projections

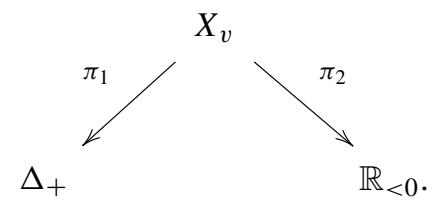

Each time a vertex, say $i \in I$, is fired, there is a natural isomorphism $X_{v} \backslash\left\{\left(\alpha_{i}, v_{i}\right)\right\} \stackrel{\sim}{\rightarrow}$ $X_{f_{i} v}$, with $(\alpha, \alpha \cdot v) \mapsto\left(s_{i} \alpha, \alpha \cdot v\right)=\left(s_{i} \alpha, s_{i} \alpha \cdot f_{i} v\right)$. The set $X_{v}$ is defined similarly in the extended Dynkin case, with $\Delta_{+}$replaced by $\widetilde{\Delta}_{+}$, and there is still a natural isomorphism $X_{v} \backslash\left\{\left(\alpha_{i}, v_{i}\right)\right\} \stackrel{\sim}{\rightarrow} X_{f_{i} v}$.

Proof In the Dynkin case, $X_{v}$ is finite. Since the size decreases by one in each step, removing an element whose second projection is the amplitude at the vertex which is fired, we see that the game is won precisely when $\pi_{2}\left(X_{v}\right) \subset[-1,0)$, and otherwise it is lost. The former is equivalent to (3.1). 
In the extended Dynkin case, the game is won precisely when $X_{v}$ is finite and $\pi_{2}\left(X_{v}\right) \subset[-1,0)$; finiteness is equivalent to $\delta \cdot v>0$. The condition $\pi_{2}\left(X_{v}\right) \subset$ $[-1,0)$ is equivalent to (3.2), and implies $\delta \cdot v \geq 0$, so for $v$ to be winning we only need to additionally assume that $\delta \cdot v \neq 0$.

Since, in the extended Dynkin case, a game that is not won is either lost or loops, it remains to show that $v$ is losing precisely when there exists $\alpha \in \widetilde{\Delta}_{+}$with $\alpha \cdot v<-1$, i.e., when $\pi_{2}\left(X_{v}\right) \not \subset[-1,0)$. It is clear that the condition is required for $v$ to be losing. Thus, suppose that $\alpha \cdot v<-1$ for some $\alpha \in \widetilde{\Delta}_{+}$. We will show that $v$ is losing. We induct on the height of $\alpha$. Suppose $v_{i}<0$, and that we fire the vertex $i$. Consider two cases: first, suppose that $h\left(s_{i} \alpha\right)<h(\alpha)$. Then, $s_{i} \alpha \cdot f_{i} v<-1$ and $h\left(s_{i} \alpha\right)<h(\alpha)$, completing the induction. Next, suppose $h\left(s_{i} \alpha\right) \geq h(\alpha)$, i.e., $s_{i} \alpha-\alpha$ is a nonnegative multiple of $\alpha_{i}$. Then, $\alpha \cdot f_{i} v \leq s_{i} \alpha \cdot f_{i} v$ (since $\left(f_{i} v\right)_{i}>0$ ), and $s_{i} \alpha \cdot f_{i} v=\alpha \cdot v$. Thus, we may leave $\alpha$ unchanged. If we eventually fire a vertex $i \in \widetilde{I}$ such that $h\left(s_{i} \alpha\right)<h(\alpha)$, the induction is complete. Otherwise, we would be playing the game only on a Dynkin subgraph, which would have to terminate in finitely many moves, and therefore reach a forbidden configuration ( since $\pi_{2}\left(X_{v}\right) \not \subset[-1,0)$ ).

Note that only finitely many inequalities in (3.2) are required: since (3.2) implies $\delta \cdot v \geq 0,(3.2)$ is equivalent to the conditions $\delta \cdot v \geq 0, \alpha \cdot v \geq-1$, and $(\delta-\alpha) \cdot v \geq-1$ for all $\alpha$ which are positive roots of a corresponding Dynkin subgraph obtained by removing an extending vertex. So, together with $\delta \cdot v \geq 0$, it is enough to assume (3.2) for $\alpha \in \Delta_{+} \cup\left(\delta-\Delta_{+}\right)$, which is finite.

Corollary 3.3 If $\delta \cdot v=0$, then the game loops (and cannot terminate) if and only if, after removing an extending vertex, both $v$ and $-v$ are winning.

Proof This follows from the fact that $\widetilde{\Delta}_{+}=\left(\Delta_{+}+\mathbb{Z}_{\geq 0} \delta\right) \sqcup\left(-\Delta_{+}+\mathbb{Z}_{>0} \delta\right)$.

Another interpretation of the above corollary is the following: $v$ continues indefinitely if and only if the restriction of $v$ to the complement of an extending vertex cannot reach a forbidden configuration by playing the numbers game forwards or backwards (i.e., firing vertices with positive instead of negative amplitudes).

For example, in Fig. 2, the configuration on the left is looping, but the one on the right is losing, despite the fact that $\delta \cdot v=0$.
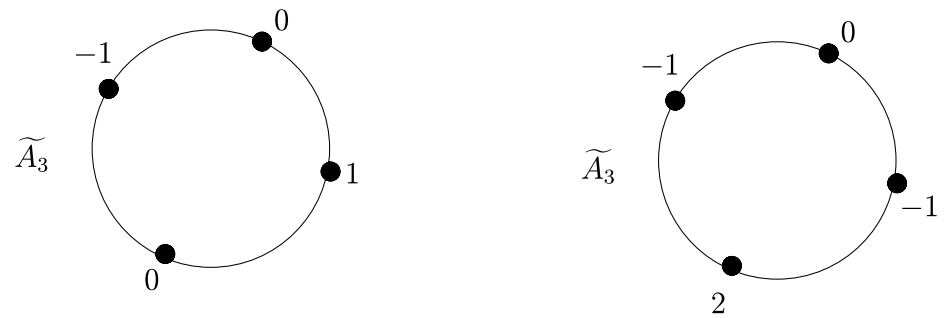

Fig. 2 Examples of a looping and a losing configuration for $\widetilde{A_{3}}$, both satisfying $\delta \cdot v=0$ 
Remark 3.4 T. Haines pointed out that Theorem 3.1 implies [14, Lemma 3.1]: for every dominant minuscule ${ }^{6}$ coweight $\mu$ and every coweight $\lambda \in W \mu$, there exists a sequence of simple roots $\alpha_{1}, \ldots, \alpha_{p}$, such that $s_{1}(\mu)=\mu-\alpha_{1}^{\vee}, s_{2} s_{1} \mu=\mu-\alpha_{1}^{\vee}-$ $\alpha_{2}^{\vee}, \ldots$, and $\lambda=s_{p} s_{p-1} \cdots s_{1}(\mu)=\mu-\alpha_{1}^{\vee}-\cdots-\alpha_{p}^{\vee}$.

\section{The integral case}

Of particular relevance is the case of integral configurations $v \in \mathbb{Z}^{I}$. Below, we apply Theorem 3.1 to give a surprisingly simple, explicit description of the losing and looping integral configurations in the Dynkin and extended Dynkin cases. (Recall that we are working only with simply-laced graphs. In the next section we study more general situations.)

To state the theorem, we will make use of the interpretation of configurations $v \in$ $\mathbb{R}^{I}$ as coweights. In particular, as in the introduction, for every Dynkin graph $\Gamma$, and every root $\alpha \in \Delta_{+}$, there is an associated coroot configuration $\alpha^{\vee} \in \mathbb{Z}^{I}$, in the basis of fundamental coweights, uniquely defined by $\beta \cdot \alpha^{\vee}=\langle\beta, \alpha\rangle$ for all $\beta$, using the Cartan form as in Sect. 2.1. For every extended Dynkin graph $\widetilde{\Gamma}$, Dynkin subgraph $\Gamma$, and $\alpha \in \widetilde{\Delta}_{+}$, we also have the configuration $\alpha^{\vee}$ defined in the same way; in particular, $\delta \cdot \alpha^{\vee}=0$ (and the $\alpha_{i}^{\vee}$ are linearly dependent). Recall that we write $\omega_{i}$ for the $i$ th fundamental coweight (in the Dynkin case), and hence for the $i$-th elementary vector in $\mathbb{Z}^{I}$ viewed as a configuration. ${ }^{7}$ Thus, $\alpha_{i} \cdot \omega_{j}=\delta_{i j}$. For $\beta \in \Delta_{+}$or $\widetilde{\Delta}_{+}$, let its support, $\operatorname{supp}(\beta)$, be the (connected) subgraph on which its coordinates $\beta_{i}$ are nonzero.

\section{Theorem 4.1}

(i) An integral configuration $v$ on a Dynkin graph is winning if and only if

(1) $v_{i} \geq-1$ for all $i$, and

(2) for all $\alpha \in \Delta_{+},\left.v\right|_{\operatorname{supp}(\alpha)} \neq-\alpha^{\vee}$;

(ii) An integral configuration $v$ on an extended Dynkin graph is winning if and only if (1) and (2) are satisfied (with $\alpha \in \widetilde{\Delta}_{+}$), and furthermore,

(3) $v \neq-\omega_{i}$ for any extending vertex $i$.

(iii) An integral configuration on an extended Dynkin graph is looping if and only if it is in the Weyl orbit of a vector $\mu=\omega_{i}-\omega_{i^{\prime}}$ for distinct extending vertices $i$, $i^{\prime}$. In this case, the numbers game can take the configuration to and from such a vector $\mu$.

Remark 4.2 The above result implies Theorem 1.3, as well as the extended Dynkin version thereof.

As in the introduction, for $\Gamma^{\prime} \subseteq \Gamma$, with vertex sets $I^{\prime} \subseteq I$, the restriction $\left.v\right|_{\Gamma^{\prime}}$ is the restriction $\mathbb{R}^{I} \rightarrow \mathbb{R}^{I^{\prime}}$ of coordinates.

\footnotetext{
${ }^{6}$ Recall that minuscule means that $\langle\mu, \alpha\rangle \in\{-1,0,1\}$ for all $\alpha \in \Delta$.

${ }^{7}$ We use distinct notation $\alpha_{i}, \omega_{i}$ for the same vector in $\mathbb{Z}^{I}$ depending on whether it is viewed as a simple root or a configuration, to avoid confusion.
} 
Fig. 3 Minimal losing configurations for $\widetilde{A_{n}}$

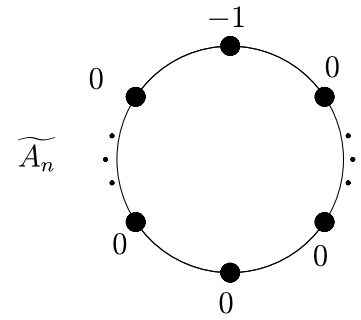

We remark that an alternative way to state parts (i) and (ii) above is that the losing configurations on (extended) Dynkin graphs which are winning on all proper subgraphs, which we call the minimal losing configurations, are exactly those of the form $-\beta^{\vee}$ for fully supported roots $\beta$ (which in the extended Dynkin case also satisfy $\beta_{i} \leq \delta_{i}$ for all $i$ ), and $-\omega_{j}$ for extending vertices $j$, together with the one-vertex forbidden configurations.

Here, we have used that $(\beta+c \delta)^{\vee}=\beta^{\vee}$ for all $c \in \mathbb{Z}$, so that in part (ii) it suffices to assume that $\beta \in \widetilde{\Delta}_{+}$satisfies $\beta_{i} \leq \delta_{i}$ for all $i$, i.e., $\beta_{i} \leq 1$ for all extending vertices $i$. In fact, we can further restrict to the case of roots $\beta$ that are supported on a Dynkin subgraph, in exchange for adding the condition that $v_{\operatorname{supp}(\gamma)} \neq \gamma^{\vee}$ for all positive roots $\gamma$ such that $\gamma_{i}=0$ at all extending vertices $i$. This is because the fully supported roots $\beta$ such that $\beta_{i} \leq \delta_{i}$ for all $i$ are exactly $\delta-\gamma$ where $\gamma \in \widetilde{\Delta}_{+}$satisfies $\gamma_{i}=0$ at all extending vertices, and then $-\beta^{\vee}=\gamma^{\vee}$.

As a special case of (ii), for $\widetilde{A_{n}}$ (with $n \geq 1$ ), the only integral losing configurations which are winning on all proper subgraphs are $-\omega_{i}$ for all $i$ (see Fig. 3). Also, by (iii), there is no looping integral configuration on $\widetilde{E_{8}}$ (but these exist for all other extended Dynkin graphs).

Proof (i) Following the discussion above, we show that the minimal losing configurations on Dynkin graphs with more than one vertex are exactly $-\beta^{\vee}$ for fully supported $\beta \in \Delta_{+}$. Note that it is clear that such configurations are minimal losing configurations, since $\beta \cdot\left(-\beta^{\vee}\right)=-2$ and $\gamma \cdot\left(-\beta^{\vee}\right) \in\{-1,0,1\}$ for all $\gamma \in \Delta_{+} \backslash\{\beta\}$. Thus, we only need to show that there are no other minimal losing configurations (other than one-vertex ones).

For any minimal losing configuration $v \in \mathbb{Z}^{I}$, Theorem 3.1 implies the existence of $\beta \in \Delta_{+}$such that $\beta \cdot v \leq-2$. By minimality, all such $\beta$ are fully supported. It suffices to prove that, when $\beta$ is not simple (i.e., the graph has more than one vertex), $v=-\beta^{\vee}$. We prove this by induction on the height of $\beta$, considering all Dynkin graphs simultaneously.

Let $i$ be a vertex such that $h\left(s_{i} \beta\right)<h(\beta)$, i.e., $\left\langle\beta, \alpha_{i}\right\rangle=1$. It follows that $v_{i}=-1$; otherwise, $s_{i} \beta \cdot v \leq-2$, a contradiction. Since $s_{i} \beta \cdot f_{i} v \leq-2$, in the case that $s_{i} \beta$ is not simple, we deduce from the inductive hypothesis that the restriction of $f_{i} v$ to the support of $s_{i} \beta$ coincides with $-\left(s_{i} \beta\right)^{\vee}$. In the case $s_{i} \beta=\alpha_{j}$ is simple, by minimality, $v_{i}=-1=v_{j}$, and hence $\left(f_{i} v\right)_{j}=-2$, which also coincides with $-\left(s_{i} \beta\right)_{j}^{\vee}$. In either case, since $-\left(\left(s_{i} \beta\right)^{\vee}\right)_{i}=\left(\beta^{\vee}\right)_{i}=1$, we deduce that $f_{i} v=-\left(s_{i} \beta\right)^{\vee}$ and hence $v=$ $-\beta^{\vee}$, as desired.

(ii) We prove that the minimal losing configurations in the extended Dynkin case are exactly $-\beta^{\vee}$ for fully supported $\beta \in \widetilde{\Delta}_{+}$satisfying $\beta_{i} \leq \delta_{i}$ for all $i$, and $-\omega_{i}$ for 
extending vertices $i$. The former configuration is a minimal losing configuration by the same argument as in the Dynkin case, and $-\omega_{i}$ is a minimal losing configuration since $\delta \cdot-\omega_{i}=-1<0$ (so $-\omega_{i}$ is losing) and $\beta \cdot-\omega_{i}=-\beta_{i} \in\{-1,0\}$ for all $\beta \in \widetilde{\Delta}_{+}$ (so $-\omega_{i}$ is winning on all Dynkin subgraphs). Hence, it suffices to prove that there are no other minimal losing configurations.

Let $v$ be an integral losing configuration which is winning on all proper subgraphs, and let $\beta \in \widetilde{\Delta}_{+}$be of minimal height such that $\beta \cdot v \leq-2$. Once again, we can induct on the height of $\beta$. We reach the desired conclusion unless $\beta=c \delta+\alpha_{i}$ for some $c \geq 1$ and $i \in \widetilde{I}$, so assume this. Since $v_{i} \geq-1$, it follows that $\delta \cdot v \leq-1$. Moreover, fix an associated Dynkin subgraph $\Gamma$. Then, for all $\gamma \in \Delta_{+}$, we must have $\gamma \cdot v \in\{-1,0\}$ (since $(\delta-\gamma) \cdot v \geq-1$ and $\gamma \cdot v \geq-1$ by minimality of $\beta$ ). In particular, $v_{j} \in\{-1,0\}$ for all $j$. In this case, in order for $v$ not to be losing on a Dynkin subgraph, we must have $v=-\omega_{i}$, where $i$ is an extending vertex.

(iii) Let $i$ be an extending vertex, and let $v \in \mathbb{Z}^{I}$ satisfy $\delta \cdot v=0$ but $v \neq 0$. If we play the numbers game by firing only vertices other than $i$, we must eventually obtain either a forbidden configuration (if the restriction of $v$ to the complement of $i$ is losing) or a configuration whose sole negative amplitude occurs at $i$. In the latter case, in order to not be forbidden, we must have -1 at the vertex $i$, and hence, in order to satisfy $\delta \cdot v=0$, there can only be one positive amplitude, it must be 1 , and it must occur at another extending vertex, say $i^{\prime}$. So, $v$ is winning when restricted to the complement of $i$ if and only if one can obtain $\mu=\omega_{i^{\prime}}-\omega_{i}$ from $v$. This implies that $v$ is in the same Weyl orbit as $\mu$. On the other hand, if $v$ is in the Weyl orbit of $\mu$, then $\delta \cdot v=0$ and the usual numbers game loops, and since $\alpha \cdot v \in\{-1,0,1\}$ for all $\alpha \in \widetilde{\Delta}_{+}$, the numbers game with a cutoff also loops. Hence, the conditions that $v$ is looping, that $v$ is in the Weyl orbit of such a $\mu$, and that $\mu$ can be obtained from $v$ by playing the numbers game with a cutoff, are all equivalent. Since, in this case, $-v$ is also looping, we see also that $-v$ can reach a configuration $v=\omega_{j}-\omega_{i^{\prime}}$ for some extending vertex $j$, and since $\nu$ is in the same Weyl orbit as $-\mu$, we must have $v=-\mu$ (since $-\mu$ and $v$ are dominant on the complement of $\left.i^{\prime}\right)$. Hence, $v$ can be obtained from $\mu$ by playing the numbers game, which proves the remainder of the final assertion.

Remark 4.3 In the Dynkin case, the above may be interpreted as saying that every losing integral configuration which is winning on all proper subgraphs is obtainable from the maximally negative coroot by playing the numbers game: this configuration is the one with $v_{i}=-1$ when $i$ is adjacent to the extending vertex of $\widetilde{\Gamma}$, and $v_{i}=0$ otherwise. On the other hand, in the non-integral case, losing configurations are not necessarily obtainable from nonpositive ones by playing the numbers game: for example, on $D_{4}$, one may place -1 at all three endpoint vertices, and $\frac{3}{2}$ at the node (Fig. 4).

Fig. 4

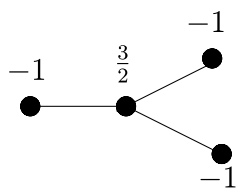


Remark 4.4 Note that the extended Dynkin case with $\delta \cdot v \geq 0$ and $v$ losing, integral, and winning on all proper subgraphs may similarly be described as those configurations obtainable from $\alpha_{i} \vee=2 \omega_{i}-\sum_{j}$ adjacent to $i \omega_{j}$, for $i$ not an extending vertex, by playing the numbers game. This contrasts with the non-integral case: see the next remark.

Remark 4.5 In the extended Dynkin case, it is perhaps surprising that all losing integral configurations with $\delta \cdot v>0$ are also losing on a proper subgraph. This is not true in the non-integral case (except in the case $\widetilde{A_{n}}$ ): e.g., one may take a configuration $\beta^{\vee}+\varepsilon \omega_{i}$, for $\beta \in \widetilde{\Delta}_{+}$which satisfies $\beta_{j}=0$ for all extending vertices $j$, and $\varepsilon \in\left(0, \frac{1}{\delta_{i}}\right)$ for any fixed $i \in \widetilde{I}$. Similarly, one may find losing configurations with $\delta \cdot v=0$ which are winning on all Dynkin subgraphs, but are not $\beta^{\vee}$ for $\beta \in \Delta_{+}$(although there are still none for $\widetilde{A_{n}}$ ): for example, $\varepsilon \beta^{\vee}$ for $\varepsilon \in\left(\frac{1}{2}, 1\right)$ and $\beta$ as before. For another example, we can take any configuration in $\widetilde{D_{n}}$ with values $a, b, c, d \geq-1$ at exterior vertices such that $\sigma:=\frac{a+b+c+d}{2}<-1$ and $\sigma-x \geq-1$ for all $x \in\{a, b, c, d\}$. Finally, there are many more losing non-integral configurations with $\delta \cdot v<0$ that are winning on all proper subgraphs than just $-\omega_{i}$ for $i$ an extending vertex: for example, $-\omega_{i}+u$ for any nonnegative vector $u$ such that $\delta \cdot u<1$

\section{Generalization to arbitrary graphs with multiplicities}

In $[10,19]$, the numbers game was stated in greater generality than the above. Namely, in addition to a graph with vertex set $I$ (and no loops or multiple edges), we are given a Coxeter group $W$ with generators $s_{i}, i \in I$ and relations $\left(s_{i} s_{j}\right)^{n_{i j}}$ for $n_{i j} \in\{1,2, \ldots\} \cup\{\infty\}$ (with $n_{i j}=1$ exactly when $i=j$ ), together with a Cartan matrix $C=\left(c_{i j}\right)_{i, j \in I}$, such that $c_{i i}=2$ for all $i, c_{i j}=0$ whenever $i$ and $j$ are not adjacent, and otherwise $c_{i j}, c_{j i}<0$ and either $c_{i j} c_{j i}=4 \cos ^{2}\left(\frac{\pi}{n_{i j}}\right)$ (when $n_{i j}$ is finite) or $c_{i j} c_{j i} \geq 4$ (when $n_{i j}=\infty$ ).

We recall that the numbers game is modified as follows in terms of $C$ : The configurations are again of the form $v \in \mathbb{R}^{I}$, and, we may fire the vertex $i$ in a configuration $v \in \mathbb{R}^{I}$ if and only if the amplitude $v_{i}<0$. The difference is that the new configuration $f_{i}\left(v_{i}\right)$ is now given by

$$
f_{i}(v)_{j}=v_{j}-c_{i j} v_{i} .
$$

We call this the weighted numbers game. The non-weighted numbers game is recovered in the case $c_{i j}=-1$ for all adjacent $i, j$.

The reflection action of $W$ on $\mathbb{R}^{I}$ is defined by

$$
s_{i}(\beta)_{j}= \begin{cases}\beta_{j}, & \text { if } j \neq i, \\ -\beta_{i}-\sum_{k \neq i} c_{i k} \beta_{k}, & \text { if } j=i .\end{cases}
$$

Recall from [10] that, in this situation, the usual numbers game is strongly convergent: if the game can terminate, then it must terminate, and in exactly the same number of moves and arriving at the same configuration, regardless of the choices made. 
We remark that, while it is standard to take $C$ to be symmetric, there are cases when this is not desired, particularly for the non-simply-laced Dynkin diagrams $\Gamma$, where $C$ can be taken to be integral only if allowed to be non-symmetric. In these cases, if we choose $C$ to be integral, playing the numbers game on $\Gamma$ is equivalent to playing the numbers game without multiplicities on a simply-laced graph $\Gamma^{\prime}$ with some symmetry group $S$, such that $\Gamma^{\prime} / S=\Gamma$, if we restrict to $S$-invariant configurations on $\Gamma^{\prime}$, where we allow simultaneous firing of any orbit of vertices under $S$ (since these orbits consist of nonadjacent vertices, it makes sense to fire them simultaneously).

Let $\Delta=\bigcup_{i \in I} W \alpha_{i}$ be the set of (real) roots. ${ }^{8}$ Let $\Delta_{+} \subset \Delta$ be the subset of positive roots: these are the elements whose entries are nonnegative. Note that, by a standard result (see, e.g., [1, Proposition 4.2.5]), $\Delta=\Delta_{+} \sqcup\left(-\Delta_{+}\right)$.

Finally, we recall a useful partial ordering from, e.g., [1, Sect. 4.6]. For $\beta \in \Delta_{+}$, we say that $\beta<s_{i} \beta$ if and only if $\beta_{i}<\left(s_{i} \beta\right)_{i}$. Generally, for $\alpha, \beta<\Delta_{+}$, we say $\alpha<\beta$ if there exists a sequence $\alpha<s_{i_{1}} \alpha<s_{i_{2}} s_{i_{1}} \alpha<\cdots<s_{i_{m}} s_{i_{m-1}} \cdots s_{i_{1}} \alpha=\beta$. The argument of [1, Lemma 4.6.2] shows that this is a graded partial ordering. The grading, $\operatorname{dp}(\alpha)$, called the depth, is defined to be the minimum number of simple reflections required to take $\alpha$ to a negative root. Thus, $\alpha<s_{i} \alpha \operatorname{implies} \operatorname{dp}\left(s_{i} \alpha\right)=$ $\operatorname{dp}(\alpha)+1$.

Theorem 5.1 Let $\Gamma, C$ be associated to a Coxeter group. Assume that $C$ satisfies $c_{i j}=c_{j i}$ whenever $n_{i j}$ is odd (and finite). Then, $v$ can reach a forbidden configuration if and only if $\beta \cdot v<-1$ for some $\beta \in \Delta_{+}$, and in this case, the minimum number of moves required to take $v$ to a forbidden configuration is

$$
m(v):=\min \left\{\operatorname{dp}(\beta)-1 \mid \beta \cdot v<-1, \beta \in \Delta_{+}\right\} .
$$

Furthermore, if $v_{i}<0$, then $m\left(f_{i} v\right) \in\{m(v), m(v)-1\}$.

Note that, in the non-simply-laced Dynkin cases with $C$ integral, we may always take $c_{i j}=c_{j i}$ whenever $n_{i j}$ is odd (and in these cases, this implies $n_{i j}=3$ ), so the theorem applies.

Corollary 5.2 Under the assumptions of the theorem, $v$ is winning if and only if the usual numbers game terminates and

$$
\alpha \cdot v \geq-1, \quad \forall \alpha \in \Delta_{+}
$$

Moreover, if (5.4) is not satisfied and the usual numbers game terminates, then $v$ is losing.

Also, under the hypotheses of the theorem, any winning configuration remains so regardless of what moves are made.

\footnotetext{
${ }^{8}$ Note that, when the Cartan matrix $C$ is associated to a nonreduced root system (i.e., $B C_{n}$ ), then $\Delta$ is a proper subset of the whole root system, which does not contain $2 \alpha$, for any simple root $\alpha$.
} 
We can also make a statement for arbitrary $C$ and $\Gamma$ :

Theorem 5.3 If $C$ and $\Gamma$ are arbitrary (associated to a Coxeter group), then $v$ can reach a forbidden configuration if and only if there exists $\beta \in \Delta_{+}$and $i \in I$ such that both $\beta \cdot v<-1$ and $\beta>\alpha_{i}$. In this case, the minimum number of moves required to reach a forbidden configuration is

$$
m^{\prime}(v):=\min \left\{\operatorname{dp}(\beta)-1 \mid \beta \cdot v<-1 \text {, and there exists } i \in I \text { with } \beta>\alpha_{i}\right\} .
$$

Moreover, in this case, if $i \in I$ is such that $v_{i}<0$, then $m^{\prime}\left(f_{i} v\right) \geq m^{\prime}(v)-1$ (provided $m^{\prime}\left(f_{i} v\right)$ is defined, i.e., $f_{i} v$ can reach a forbidden configuration).

The difference from Theorem 5.1 is that we added the condition $\beta>\alpha_{i}$, and replaced the equality for $m$ under numbers game moves by an inequality.

We remark that the usual numbers game, beginning with $v$, terminates if and only if

$$
\# \mathbb{P}\left\{\beta \in \Delta_{+} \mid \beta \cdot v<0\right\}<\infty,
$$

for arbitrary $\Gamma, C$, where $\mathbb{P}$ means modding by nonzero scalar multiplication, since each move decreases the size of this set by one. (We do not need to mod by scalar multiples if $c_{i j}=c_{j i}$ whenever $n_{i j}$ is odd.) So, this gives a completely root-theoretic description of the winning conditions above. ${ }^{9}$

For the finite and affine cases, we have the following corollary, which generalizes Theorem 3.1. As before, in the affine case, let $\delta \in \mathbb{R}_{>0}^{I}$ be the additive generator of the semigroup $\left\{\delta^{\prime} \in \mathbb{R}_{>0}^{I} \mid \alpha \in \Delta_{+} \Rightarrow \alpha+\delta^{\prime} \in \Delta_{+}\right\}$. In particular, $\langle\delta, \alpha\rangle=0$ for all $\alpha \in \Delta$.

Corollary 5.4 Let $\Gamma, C$ be associated to a finite or affine Coxeter group and let $v$ be a nonzero configuration. Then, exactly one of the following is true:

(a) (5.4) is satisfied, and $\delta \cdot v \neq 0$ or the Coxeter group is finite: then $v$ is winning, and cannot reach a forbidden configuration.

(b) (5.4) is satisfied but $\delta \cdot v=0$ : then $v$ is looping, and cannot reach a forbidden configuration.

(c) (5.4) is not satisfied. Then, provided $c_{i j}=c_{j i}$ whenever $n_{i j}$ is odd, $v$ is losing.

Note that, by Theorem 5.3, we can strengthen this slightly by replacing (5.4) by the condition that $\alpha \cdot v \geq-1$ only for $\alpha$ such that $\alpha>\alpha_{i}$ for some $i \in I$.

Proof of Corollary 5.4 (a) In the affine case, $\delta \cdot v>0$, so in either case, the usual numbers game terminates. Then, $v$ is winning by Theorem 5.3, and a forbidden configuration cannot be reached.

\footnotetext{
${ }^{9}$ Also, this observation easily implies the main results (Theorems 2.1 and 4.1) of [4]: if $v_{i} \leq 0$ for all $i$ and $v \neq 0$, then the usual numbers game can only terminate if $\Gamma, C$ are associated to a finite Coxeter group: otherwise (assuming $\Gamma$ is connected), infinitely many elements $\beta \in \Delta_{+}$which are not multiples of each other satisfy $\beta \cdot v<0$ : note that, for each $i \in I$, the set $\mathbb{P}\left(W \alpha_{i}\right)$ essentially does not depend on the choice of $C$ for a given Coxeter group.
} 
(b) $v$ is looping, as in the simply-laced case, since the usual numbers game cannot terminate, and the configuration is uniquely determined by its restriction to a subgraph obtained by removing an extending vertex, where the configuration remains in the orbit of the restriction of $v$ under the associated finite Coxeter group. The rest follows from Theorem 5.3.

(c) In this case (we assume $c_{i j}=c_{j i}$ whenever $n_{i j}$ is odd), $v$ can reach a forbidden configuration. Moreover, in the proof of Theorem 5.1, we see that there always exists a vertex $i \in I$ so that, for any configuration $v^{\prime}$ obtained from $v$ by firing vertices other than $i$, we have $m\left(f_{i} v^{\prime}\right)=m\left(v^{\prime}\right)-1$. In the affine Coxeter group case, in order for the numbers game to continue indefinitely, all vertices must be fired infinitely many times. This proves the result.

Remark 5.5 The weakened conclusions of Theorem 5.3 are needed. Indeed, if $c_{i j} \neq c_{j i}$ for some $i, j$ with $n_{i j}$ odd, then it is possible that a winning configuration can become a losing one. For example, take $I=\{1,2\}$ and $C=\left(\begin{array}{cc}2 & -2 \\ -\frac{1}{2} & 2\end{array}\right)$, with $n_{12}=3$. Then, the configuration $\left(-\frac{1}{2},-\frac{1}{2}\right)$ is winning under the sequence $\left(-\frac{1}{2},-\frac{1}{2}\right) \mapsto\left(-\frac{3}{4}, \frac{1}{2}\right) \mapsto\left(\frac{3}{4},-1\right) \mapsto\left(\frac{1}{2}, 1\right)$, but if we instead fired vertex 1 first, we would get $\left(\frac{1}{2},-\frac{3}{2}\right)$, which is forbidden.

Remark 5.6 It is natural to ask what can happen in the numbers game with a cutoff if it continues indefinitely. Suppose this happens, and let $\Gamma^{\prime}$ be the subgraph on vertices which are fired infinitely many times. If $\Gamma^{\prime}$ corresponds to an affine Coxeter group, then the configuration restricted to $\Gamma^{\prime}$ is looping, and in this case, in order for a forbidden configuration not to be reached, $\Gamma^{\prime}$ must be the whole graph (assuming that our whole graph is connected). Otherwise, if our graph is not affine, then $\Gamma^{\prime}$ cannot be associated to an affine or finite Coxeter group. Then, for any affine subgraph $\Gamma_{0} \subseteq \Gamma^{\prime}$ (where by this we allow reducing the numbers $n_{i j}$ for edges between vertices of $\Gamma_{0}$ ), the dot product of the restriction of $v$ with the associated $\delta_{0}$ must remain positive, and the value must be decreasing. It must converge to some nonnegative number, and hence all amplitudes of vertices in $\Gamma^{\prime}$ must converge to zero. In particular, the configuration $v$ must converge to some limiting allowed configuration (which is zero on $\Gamma^{\prime}$ ), and one could continue the numbers game from this limit if desired. Note that, in the case that $c_{i j}=c_{j i}$ for all odd $n_{i j}$, we must also have $\alpha \cdot v>-1$ for all $\alpha \in \Delta_{+}$supported on $\Gamma^{\prime}$, i.e., $\left.v\right|_{\Gamma^{\prime}}$ cannot reach a forbidden configuration by playing the numbers game on $\Gamma^{\prime}$.

\subsection{Proof of Theorems 5.1 and 5.3}

We will use the following lemma which is interesting in itself (and is the connection between the two theorems):

Lemma 5.7 If $\Gamma, C$ are such that $c_{i j}=c_{j i}$ whenever $n_{i j}$ is odd, then for all $\beta \in \Delta_{+}$, we have $\alpha_{i} \leq \beta$ for some $i \in I$.

We remark that it is well known (and obvious) that the lemma holds when $C$ is symmetric. 
Proof The case $n_{i j}$ is odd is exactly the case when, on the subgraph with vertices $i$ and $j$ only, $\alpha_{i}$ is in the $W$-orbit of some positive multiple of $\alpha_{j}$ and vice-versa (and this multiple is 1 if and only if $c_{i j}=c_{j i}$ ). Thus, this assumption is exactly what is needed so that, whenever $\beta=a \alpha_{i}+b \alpha_{j} \in \Delta_{+}$and $d \alpha_{i}<\beta$ for some $d \in \mathbb{R}$, then $d=1$. As a result, using the Coxeter relations, it follows inductively on depth that, if $\alpha_{i}<\beta$ for some $i \in I$, then if $\gamma<\beta$ and $\gamma \in \Delta_{+}$is not simple, we also have $\alpha_{j}<\gamma$ for some $j \in I$. Thus, for all $\beta \in \Delta_{+}$, there exists $i \in I$ with $\alpha_{i} \leq \beta$.

Proof of Theorem 5.1 It will be convenient to think of $m(v)$ as being allowed to be infinite (infinite if and only if the set appearing in the right hand side is empty). Similarly, call the number of moves required to reach a forbidden configuration "infinite" if and only if a forbidden configuration cannot be reached. We clearly have $m(v) \geq 0$, and Lemma 5.7 implies that $m(v)=0$ if and only if $v$ is forbidden. Thus, using induction, the theorem may be restated as: if $v$ is not forbidden, then for any vertex $i$ with $v_{i}<0$, we have $m\left(f_{i} v\right) \in\{m(v), m(v)-1\}$, and there exists at least one such $i$ with $m\left(f_{i} v\right)=m(v)-1$. Here, $\infty+c:=\infty$ for any finite $c$.

Suppose that $\alpha \in \Delta_{+}$and $j \in I$ are such that $\alpha \cdot v<-1$ and $v_{j}<0$. If we fire $j$, then the set $\left\{\beta \in \Delta_{+}: \beta \cdot v<-1\right\}$ changes by applying $s_{j}$ and intersecting with $\Delta_{+}$. Hence, $m\left(f_{j} v\right) \in\{m(v)-1, m(v), m(v)+1\}$. In particular, $m\left(f_{j} v\right) \geq m(v)-1$.

Suppose that $\alpha \in \Delta_{+}$is such that $\alpha \cdot v<-1$ and $\operatorname{dp}(\alpha)-1=m(v)$, and let $i \in I$ be such that $s_{i} \alpha<\alpha$. Then, if $v_{i} \geq 0$, then $s_{i} \alpha \cdot v \leq \alpha \cdot v<-1$, which would contradict the minimality of the depth of $\alpha$. Thus, $v_{i}<0$, and it follows that $m\left(f_{i} v\right)=m(v)-1$. So, there exists $i$ such that $m\left(f_{i} v\right)=m(v)-1$.

Next, suppose that $v_{i}<0$ and $s_{i} \alpha>\alpha$. Then, $\alpha \cdot f_{i} v \leq s_{i} \alpha \cdot f_{i} v<-1$. As a result, we have $m\left(f_{i} v\right) \in\{m(v), m(v)-1\}$. Thus, for any $i \in I$ such that $v_{i}<0$, we have $m\left(f_{i} v\right) \in\{m(v), m(v)-1\}$.

Proof of Theorem 5.3 This is similar to the proof of Theorem 5.1. Define the set

$$
Y_{v}:=\left\{\beta \in \Delta_{+}: \beta \cdot v<-1 \text { and } \beta>\alpha_{i} \text { for some } i\right\} .
$$

We need to show that, whenever $v_{j}<0$, the minimum depth of an element of $Y_{f_{j} v}$ is at most one less than that of $Y_{v}$, and that we can achieve exactly one less by picking $j$ appropriately. We first prove the inequality. Let $\alpha \in Y_{f_{j} v}$ be an element of minimal depth. If $s_{j} \alpha>\alpha$, then $s_{j} \alpha \in Y_{v}$ and the statement follows. If $s_{j} \alpha<\alpha$, then $v_{j}<0$ implies that $\alpha \in Y_{v}$, and the statement follows. Next, for the equality, let $\alpha \in Y_{v}$ be an element of minimal depth. It suffices to show that there exists $j$ such that $s_{j} \alpha<\alpha$ and $s_{j} \alpha \in Y_{f_{j} v}$. For this, it suffices to choose $j$ so that there exists $i$ such that $s_{j} \alpha<\alpha$ and $\alpha_{i}<s_{j} \alpha$. This exists by definition.

Remark 5.8 Note that, as a corollary of Lemma 5.7, we see that, for a general Coxeter group $W$, vertex $i \in I$, and matrix $C$, the set $\left\{j \in I \mid \exists b \in \mathbb{R}, b \alpha_{j} \in W \alpha_{i}\right\}$ is the set of vertices $j$ connected to $i$ by a sequence of edges $i^{\prime} \mapsto j^{\prime}$ corresponding to odd integers $n_{i^{\prime}, j^{\prime}}$. It is clear that all such $j$ are in the set; conversely, if an edge corresponding to an even integer or $\infty$ is required to connect $i$ to $j$, then if $w \alpha_{i}=$ $b \alpha_{j}$, then by modifying the elements of $C$ corresponding to the edges with even or infinite $n_{i^{\prime} j^{\prime}}$, we would be able to change the value $b$ such that $b \alpha_{j} \in W \alpha_{i}$. But this is 
impossible, since $b=1$ whenever $c_{i^{\prime} j^{\prime}}=c_{j^{\prime} i^{\prime}}$ for all odd $n_{i^{\prime} j^{\prime}}$, and symmetrizing the latter values of $C$ would rescale $b$ by a fixed amount independent of the other values of $C$ (and independent of $b$ itself).

Acknowledgements We thank R. Kottwitz for useful comments and M. Boyarchenko for the opportunity to speak on the topic. The first author is an EPDI fellow and the second author is an AIM fellow, and both authors were supported by Clay Liftoff fellowships. The first author was also partially supported by the EPSERC Grant EP/F005431/1, and the second author was partially supported by the University of Chicago's VIGRE grant. We thank the University of Chicago, MIT, the Max-Planck Institute in Bonn, and the Isaac Newton Institute for Mathematical Sciences, for hospitality.

\section{References}

1. Björner, A., Brenti, F.: Combinatorics of Coxeter groups. Springer, New York (2005)

2. Bourbaki, N.: Elements of Mathematics, Lie Groups and Lie Algebras, Chap. 4-6. Springer, Berlin Heidelberg (2002)

3. Broer, A.: Line bundles on the cotangent bundle of the flag variety. Invent. Math. 113, 1-20 (1993)

4. Donnelly, R.G., Eriksson, K.: The numbers game and Dynkin diagram classification results (2008). arXiv:0810.5371

5. Eriksson, K.: Convergence of Mozes' game of numbers. Linear Algebra Appl. 166, 151-165 (1992)

6. Eriksson, K.: Strongly convergent games and Coxeter groups, Ph.D. thesis, KTH, Stockholm (1993)

7. Eriksson, K.: Node firing games on graphs. In: Jerusalem Combinatorics '93: An International Conference in Combinatorics (May 9-17, 1993, Jerusalem, Israel), vol. 178, pp. 117-128. Am. Math. Soc., Providence (1994)

8. Eriksson, K.: Reachability is decidable in the numbers game. Theor. Comput. Sci. 131, 431-439 (1994)

9. Eriksson, K.: The numbers game and Coxeter groups. Discrete Math. 139, 155-166 (1995)

10. Eriksson, K.: Strong convergence and a game of numbers. Eur. J. Comb. 17(4), 379-390) (1996)

11. Gashi, Q.R.: The conjecture of Kottwitz and Rapoport in the case of split groups, Ph.D. thesis, The University of Chicago (2008)

12. Gashi, Q.R.: On a Conjecture of Kottwitz and Rapoport, Ann. Sci. d'E.N.S. (2010, to appear). arXiv:0805.4575v2

13. Gashi, Q.R., Schedler, T., Speyer, D.: Looping of the numbers game and the alcoved hypercube (2009). arXiv:0909.5324v1

14. Haines, T.J.: Test functions for Shimura varieties: the Drinfeld case. Duke Math. J. 106(1), 19-40 (2001)

15. Kottwitz, R.E.: On the Hodge-Newton decomposition for split groups. Int. Math. Res. Not. 26, 1433$1447(2003)$

16. Kottwitz, R.E., Rapoport, M.: On the existence of $F$-isocrystals. Comment. Math. Helv. 78, 153-184 (2003)

17. Mazur, B.: Frobenius and the Hodge filtration. Bull. Am. Math. Soc. 78, 653-667 (1972)

18. Mazur, B.: Frobenius and the Hodge filtration (estimates). Ann. Math. (2) 98, 58-95 (1973)

19. Mozes, S.: Reflection processes on graphs and Weyl groups. J. Comb. Theory, Ser. A 53(1), 128-142 (1990)

20. Proctor, R.A.: Bruhat lattices, plane partition generating functions, and minuscule representations. Eur. J. Comb. 5, 331-350 (1984)

21. Proctor, R.A.: Minuscule elements of Weyl groups, the numbers game, and $d$-complete posets. J. Algebra 213, 272-303 (1999)

22. Stembridge, J.R.: Minuscule elements of Weyl groups. J. Algebra 235(2), 722-743 (2001)

23. Wildberger, N.J.: A combinatorial construction for simply-laced Lie algebras. Adv. Appl. Math. 30, 385-396 (2003)

24. Wildberger, N.J.: Minuscule posets from neighbourly graph sequences. Eur. J. Comb. 24, 741-757 (2003) 